\title{
Design and development of automated seeding and irrigation system
}

\author{
Anirban Kumar ${ }^{1}$, Heshalini Rajagopal ${ }^{1}$ \\ ${ }^{1}$ Department of Electrical and Electronic Engineering, Manipal International University, Malaysia \\ Ianirban_kumar1993@yahoo.com
}

\begin{abstract}
Malaysia, has a very conducive environment for agriculture. Six percent of the population are employed to some of the agriculture activities making agriculture the most curtail industry in Malaysia. Hence, there is a need to develop the agricultural facilities by incorporating latest technological advancements. Conventional seeding process is time consuming and requires additional labor cost. In this paper, an automated system is proposed for seeding as well as irrigation process in agriculture which reduces the labor cost. This system aims to increase the efficiency of the seeding process without affecting the nature of soil. The proposed system is equipped with Arduino MEGA and Arduino UNO which acts as the main control unit while ultrasonic and soil moisture sensors are used to detect the obstacles and soil moisture level, respectively. The robot consists of a funnel like arrangement to perform the seeding procedure. The field is equipped with moisture sensors placed at different areas that monitors the moisture level of the soil on a regular interval for irrigation purposes. The proposed system will be of great benefit to the future endeavor of agricultural business as well as it will be able to optimize the seeding and irrigation.
\end{abstract}

Keywords: Agriculture, Seeding, Irrigation, Arduino MEGA, Ultrasonic sensors, Soil moisture sensors.

\section{Introduction}

Agriculture is the oldest and yet an important economic activity for any given country. There are distinct available types of machines for harvesting, ploughing, pesticide control etc. Most of these machines are manually operated to execute the required action. Different machines are used to perform separate action which leads to lower profit returns [1]. Seeding is a tedious yet a crucial activity for a farmer. Seeding of a large farmland is even more tedious, which demands for big group of personnel for manual seeding. To minimize the personnel required for seeding, harvesting etc., agriculture machineries were developed. Planting of seeds manually, leads to low spacing efficiency and seed placement, serious health issues and tedious task [2]. Ideal solution to overcome the deficiencies discussed above is by developing a machine that carries out more than one action and by atomization of the developed machine, which will lead to intensification of the yield.

The primary aim of this paper is to design and develop an efficient seeding and irrigation system. This system consists of an Arduino MEGA, Arduino UNO, ultrasonic sensors, DC motor, servo motors and moisture sensors. The scissor lift is designed using Fusion 360 and 3D printed. Arduino MEGA and UNO holds all the commands, receive all the parameters from the sensors and send output signals accordingly. The ultrasonic sensors will be used for the object detection and moisture and DHT 22 sensor will continuously monitor the moisture of the soil, humidity and temperature of the surroundings on a regular interval and alerts the monitoring system.

\section{Prior Work}

M.Aravind Kumar et al. (2018) have proposed a machine for seeding and irrigation to limit the working expense and decrease the ideal opportunity for planting seed \& irrigation operations by using solar energy to operate the system. There is a sharp pointed iron attached to the front of the robot to dig the soil for seed sowing, once the seed is planted the supporter at the back end of the robot covers the dug land with soil. Versatile computerized water system robot was built to irrigate the plants appropriately [3]. However, this study does not irrigate the planted seed immediately after the seeding process is completed.

Md. Didarul Islam Sujon et al. (2018) have proposed an autonomous prototype robot that will help farmers in the farmland. It is an Arduino controlled robot that carries out ploughing, sowing and watering process. The robot carries out farming using ultrasonic sensors in order to change its position from one farming strip to another. The robot aims contribute greatly in developing the farming strategies and reduce farmers cost [4]. However, this robot can carry out farming for land area of limited land area only.

K.A.Sunitha et al. (2017) have proposed a robotic system that localizes the path and can navigate itself without human action. For ploughing, this robot is equipped with tentacles that has saw blades. The locomotion is provided with wheels covered under conveyor belts. For each rotation every tooth on gear will take seeds and will drop them on field. Camera at the front end tracks the path for every fixed distance and at the minimum distance it takes the path pre-programmed [5]. However, this study has a drawback where the system 
uses camera and image processing for object detection which makes the system complex. In addition, the object detection can be inaccurate if the image captured is subjected to motion blur due to the dirty camera lens and camera movement.

S. Thawali et al. (2017) have proposed robot capable of performing operations like automatic ploughing, seed dispensing and pesticide spraying autonomously and also provides manual control when required. Initially the robot digs the entire field simultaneously dispensing seeds side by side. On the field the robot operates on automated mode. For manual control the robot uses the Remote controller as control device and helps in the navigation of the robot on the field [6]. However, there is no obstacle detection system and hence there are chances that this robot will runover planted plats if there is no human presence to deviate the robot from the predefined path.

Thorat Swapnil V et al. (2017) have proposed a sowing machine which is operated. This machine is capable of planting different types and different sizes of seeds and also the spacing between two seeds can be varied. Seed storage tank is connected at the top of the robot near rear wheels. Front sensor serves the function of guiding the robot. As any obstacle comes in front of robot it gives the signal to the robot and diverts the path of robot [7]. However, the system does not monitor the soil or plant conditions so the farmers still have to invest in systems that monitor those two variables.

\section{Methodology}

Fig 1 shows the block diagram for the motion, seeding, irrigation, obstacle detection and avoidance. The main control unit of this system is Arduino MEGA which is powered by a $9 \mathrm{~V}$ battery. Ultrasonic sensors will give input to the Arduino MEGA regarding the obstacle detection. Arduino MEGA will analyse the received inputs and control the output to the lifting servos. DC motors are to be powered by a 7.7V Lithium Polymer battery; the motors are used for the movement of the robot. Arduino MEGA controls the motor direction and speed (RPM) using L298N module.

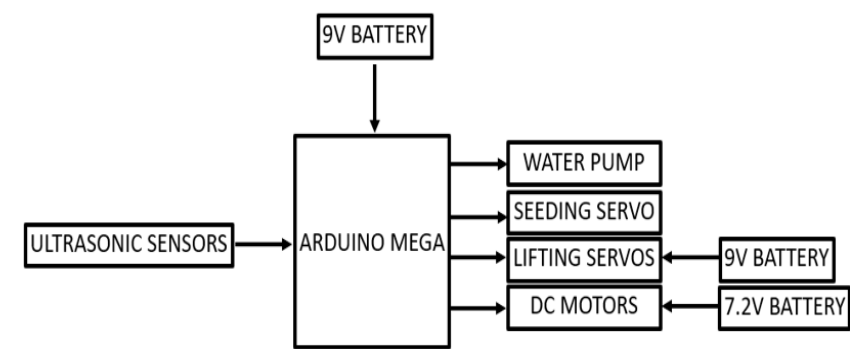

Fig. 1: Block Diagram for Seeding, Irrigation and Obstacle Avoidance

Fig. 2 shows the block diagram for the soil moisture, humidity and temperature monitoring system. Soil moisture and DHT 22 sensors will give input to the Arduino UNO regarding the soil moisture level, temperature and humidity of the surrounding respectively. The input information from the sensors will be sent to the Android Application and SMS via GSM module.

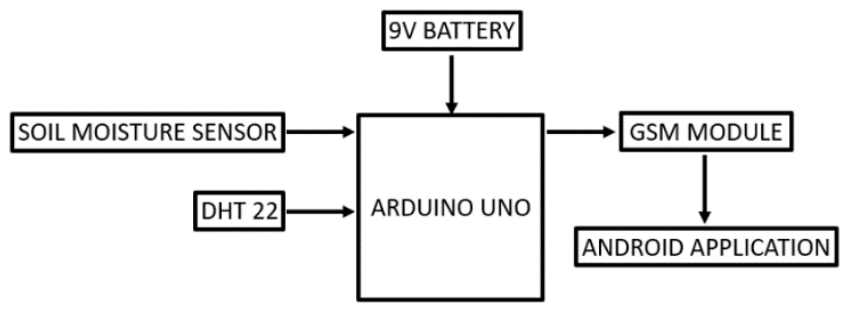

Fig. 2: Block Diagram for Soil Moisture, Humidity and Temperature Monitoring System

\subsection{Circuit Diagram and Simulation}

\subsubsection{Arduino DC Motor Control}

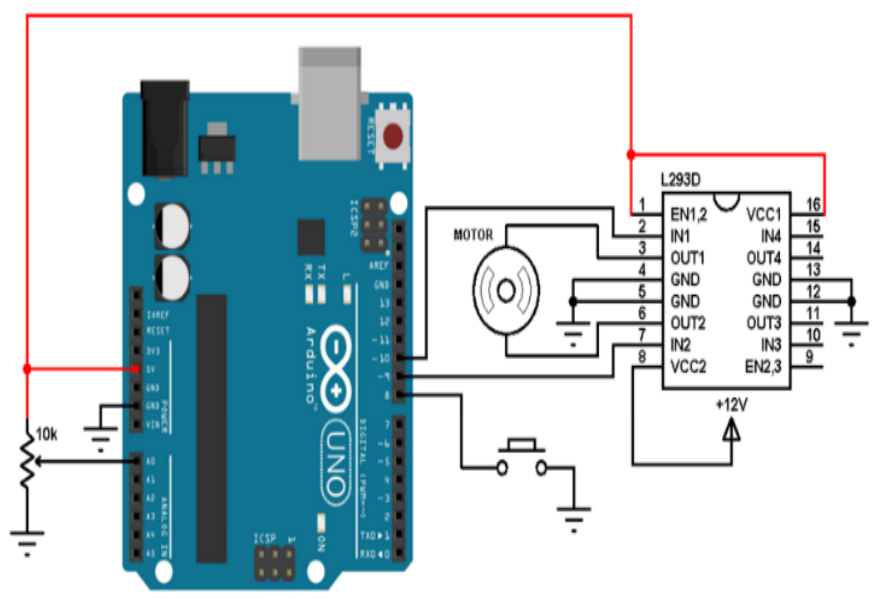

Fig. 1: Arduino DC Motor Control

Fig. 3 shows the circuit diagram of Arduino DC motor control. Only three Arduino pins are required, pin 8 is for the push button which controls the direction of motor rotation. Pins 9 and 10 are PWM signal outputs, at a given time there is only one active PWM, this allows for the control of direction as well as the speed by varying the duty cycle of the PWM signal. The active PWM pin decides the motor direction of rotation (one at a time, the other output is logic 0 ). The speed of the DC motor (both directions) is controlled with the $10 \mathrm{k} \Omega$ potentiometer which is connected to analog channel 0 (A0). The L293D driver has 2 VCCs: VCC1 is $+5 \mathrm{~V}$ and $\mathrm{VCC} 2$ is $+12 \mathrm{~V}$. Pins IN1 and IN2 are the control pins and their functions are shown in Table 1.

\begin{tabular}{|c|c|c|}
\hline IN1 & IN2 & Function \\
\hline L & H & Direction 1 \\
\hline H & L & Direction 2 \\
\hline L & L & Fast motor stop \\
\hline H & H & Fast motor stop \\
\hline
\end{tabular}

Table 1: IN1 and IN2 function 


\subsubsection{Lifting System}

Fig. 4 shows the circuit diagram for the lifting system that uses ultrasonic sensor, Arduino and motor.

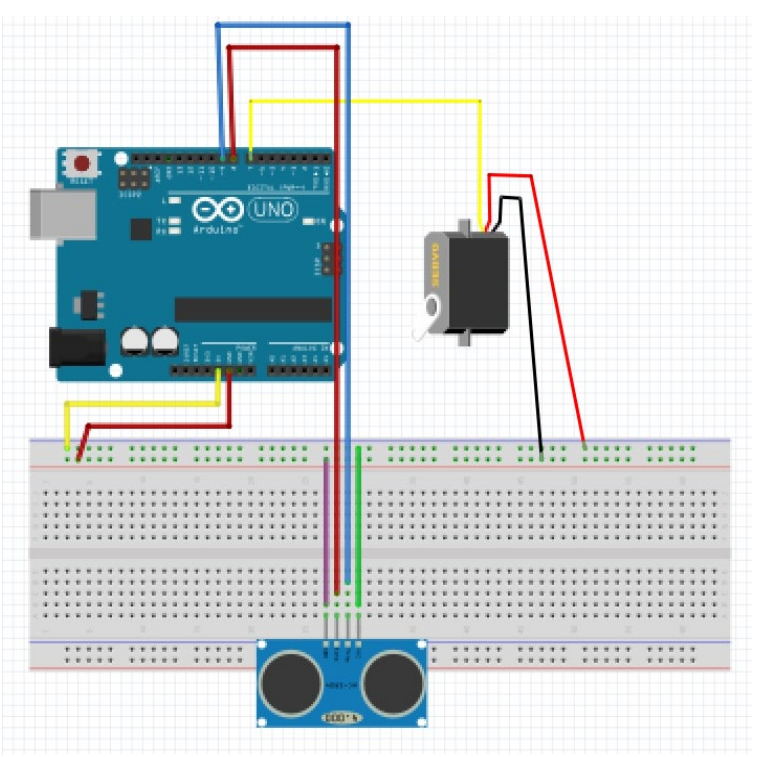

Fig 4: Lifting System

The lift like system will be used to propel the robot upwards once the ultrasonic sensor detects any obstacle, the system will be propelled upwards to a predefined height. Once the robot reaches the predefined height, the plant will be irrigated and then the robot will move forward to irrigate the plants ahead. When all the plants in that segment are irrigated the robot will be brought down.

\subsection{Flow Chart}

The various steps undertaken to develop the code to operate the proposed system is shown in Fig 5. Once the hardware design and assembly of the seeding and irrigation robot is completed, code is developed to carry out the desired action. During the coding process mainly loops like "while", "if" and "for" will be used to achieve the desired movements.

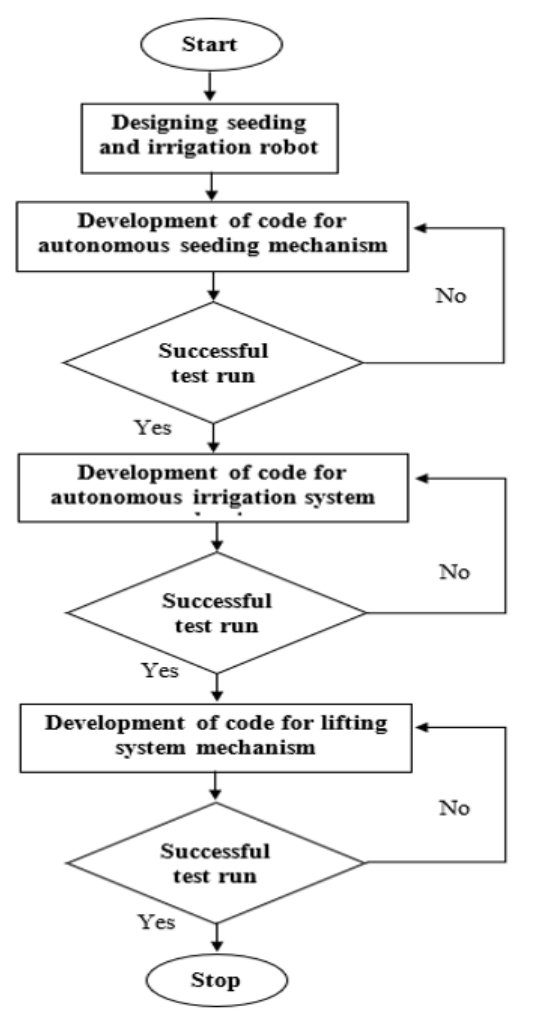

Fig. 5: Flowchart for Software Development

\section{Results}

\subsection{Seeding Mechanism}

The first objective of the system is to design an automated robot to perform seeding and irrigation procedure. The robot takes ten seconds to initialize. After initialization robot moves forward for two seconds at the speed of $200 \mathrm{rpm}$ and then comes to a halt to facilitate the seeding and irrigation process, respectively. Once the seeding and irrigation process is completed, the robot moves forward for two more times before it turns to the next row. The robot is programmed to drops the seed at an interval of six seconds and $7 \mathrm{~cm}$. Fig. 6 shows the seeding process of mung dhal 


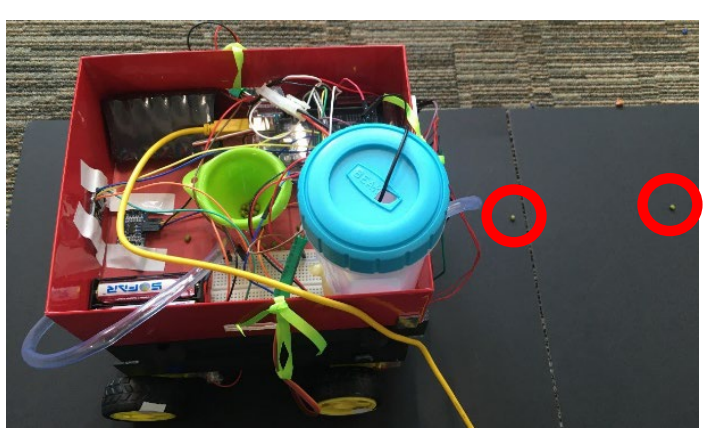

Fig. 6: Seeding Process of Mung Dhal

\subsection{Irrigation Mechanism}

Fig. 7 shows that the robot performs irrigation to the pla nt after the seeding process. For the design of water harvesti ng systems, it is necessary to assess the water requirement o $f$ the crop intended to be grown. For crops like mung dhal and peas initially $20 \mathrm{ml}$ to $25 \mathrm{ml}$ water is required for irrigat ing each seed. Table 2 shows the irrigation details of the rob ot prototype.

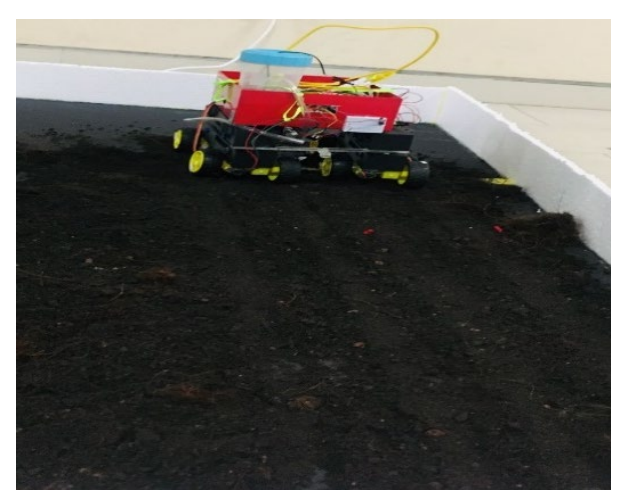

Fig. 7: Robot performing irrigation

Table 2: Irrigation Detail

\begin{tabular}{|l|}
\hline Irrigation Tank Capacity: $500 \mathrm{ml}$ \\
\hline Irrigation Pump Flow Rate: $1.2-1.6 \mathrm{~L} / \mathrm{min}$ \\
\hline Irrigation per seed: $20 \mathrm{ml}-30 \mathrm{ml}$ \\
\hline Irrigation type: Drip irrigation \\
\hline
\end{tabular}

\subsection{Monitoring System}

The second objective of the study is to develop a temperature and soil moisture monitoring system by using Android Application to enhance the user accessibility. The soil moisture, humidity and temperature monitoring system is in place for the landowner or the user to monitor the soil conditions of the separate segments using the Android App which was created using the MIT App Inventor. The app is designed to display the information regarding the soil moisture, humidity and temperature level of each land segment. The layout of the App is shown in Fig. 8.

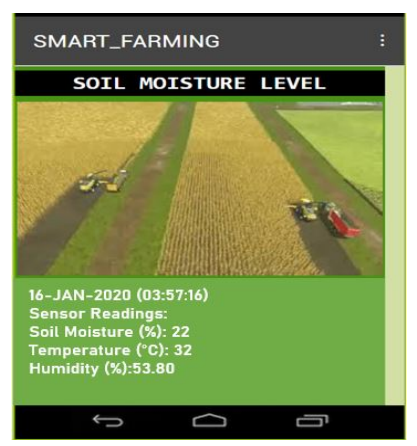

Fig. 8: Monitoring the Soil Condition Using Android App

The monitoring system is beneficial for the user, because it helps to keep track of the soil moisture, the surrounding environment and carry out on demand irrigation. It also helps the farmers to store the data for future weather prediction and planning on land management based on the data collected.

\subsection{Lifting System}

Thirdly, this paper aimed to design and develop a lifting system to avoid obstacles while maneuvering and assist in irrigation. The robot is equipped with two ultrasonic sensors to detect obstacles at the front and base of the robot. When front ultrasonic sensor detects an obstacle at distance of 10 $\mathrm{cm}$ or less, the system stops and activates the lifting system to avoid the obstacles. When the system has fully lift upwards, the base ultrasonic sensor activates, and the robot continues to move forward. The base ultrasonic sensor is placed in order to bring the lifted system back to its original level if distance between the lifted system and ground is more than $20 \mathrm{~cm}$ and if there is no obstacle in the front. Fig. 9 shows that the robot is lifted when there is an obstacle in front. 


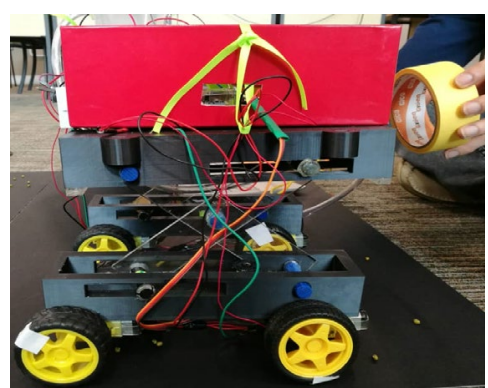

Fig. 9: Robot Lifts Up

The robot is capable to run for about $4 \mathrm{~km}$ in a single battery charge.

\section{Conclusions}

The proposed system is capable to perform seeding followed by irrigation procedure. The robot is equipped with eight wheels to facilitate the movement of the robot. The robot is also equipped with two ultrasonic sensors of any obstacle at the front and base of the robot to detect obstacles in the front and under the robot. The robot lifts up before moving forward when it detected obstacles in the front and comes back down with the assistance of the base ultrasonic sensor when the obstacle has been passed. This also enables the system to water the tall plants. Soil moisture and DHT22 sensors are fixed on the land to sense the soil moisture, humidity and temperature. The readings of these parameters are sent to the user vis SMS every one minute. In addition, the user can view the readings of the parameters via Android App. The robot moves forward once and perform the seeding at one point, irrigate the seed and moves to the next point for the seeding process. The robot dispenses seeds automatically at a fixed distance $(7 \mathrm{~cm})$.

\section{References}

[1] A. A. M. M. Amrita Sneha.A, "Agricultural Robot for Automatic Ploughing and Seeding," IEEE International Conference on Technological Innovations in ICT for Agriculture and Rural Development, pp. 17 - 23, 2015.

[2] M. L. K. P. G. V. P. R. N. Thorat Swapnil V, "Design and Fabrication of Seed Sowing Machine," International Research Journal of Engineering and Technology (IRJET), vol. 04, no. 09, pp. 704 - 707, 2017.

[3] M. Kumar, A. S. Reddy and K. Sagadevan, "Automatic Seed Sowing \& Irrigation Agribot using Arduino," International Journal of Pure and Applied Mathematics, vol. 119, no. 14, pp. 1089-1093, 2018.

[4] M. D. I. Sujon, R. Nasir, M. M. I. Habib, M. I. Nomaan, J. Baidya and M. R. Islam, "Agribot: Arduino Controlled Autonomous Multi-Purpose Farm Machinery Robot for Small to Medium Scale Cultivation," International Conference on Intelligent Autonomous Systems, pp. 155-159, 2018.

[5] K. A. Sunitha, G. S. G. S. Suraj, C. P. N. Sowrya, G. A. Sriram, D. Shreyas and T. Srinivas, "Agricultural Robot
Designed for Seeding Mechanism," IOP Conference Series: Materials Science and Engineering, 2017.

[6] S. Thawali, B. Yadav, H. Rumde and R. S. Sewane, "Design and Manufacturing of Robot for Digging and Seeding in Agriculture," International Conference on Ideas, Impact and Innovation in Mechanical Engineering, vol. 5, no. 6, p. 1637 - 1644, 2017.

[7] M. L. K. P. G. V. P. R. N. Thorat Swapnil V, "Design and Fabrication of Seed Sowing Machine," International Research Journal of Engineering and Technology, vol. 4, no. 9, pp. 704-707, 2017. 